\title{
Apprendre le français par le provençal : l'échec du frère Savinian
}

\section{Pierre Boutan}

\section{Q OpenEdition}

1 Journals

Édition électronique

URL : http://journals.openedition.org/trema/1519

DOI : 10.4000/trema.1519

ISSN : 2107-0997

\section{Éditeur}

Faculté d'Éducation de l'université de Montpellier

\section{Édition imprimée}

Date de publication : 1 septembre 2003

Pagination : $6-28$

ISSN : 1167-315X

\section{Référence électronique}

Pierre Boutan, «Apprendre le français par le provençal : l'échec du frère Savinian », Tréma [En ligne], 22 | 2003, mis en ligne le 01 septembre 2003, consulté le 19 avril 2019. URL : http:// journals.openedition.org/trema/1519; DOI : 10.4000/trema.1519

Ce document a été généré automatiquement le 19 avril 2019.

Trema 


\title{
Apprendre le français par le provençal : l'échec du frère Savinian
}

\author{
Pierre Boutan
}

La « renaissance » de la langue d'oc engagée par le Félibrige autour de Mistral dans la deuxième moitié du XIX ${ }^{\mathrm{e}}$ siècle a suscité une innovation pédagogique notoire grâce au frère des Écoles chrétiennes Savinien (Savinian en provençal). De son vrai nom Joseph Lhermite (1844-1920), il a enseigné dans le Gard puis à Arles et surtout à Avignon, où il devient directeur de l'école puis inspecteur de la province. Pour définir sommairement son innovation, on peut utiliser une citation du frère Savinian lui-même : «Dans le Midi de la France surtout, on voit peu d'élèves qui puissent, à la fin de leurs études primaires, se servir convenablement de la langue écrite ou parlée. La principale cause de cette impuissance est dans l'absence des études comparatives. $"{ }^{1}$. Après avoir rappelé quelques données de base sur l'histoire de l'enseignement

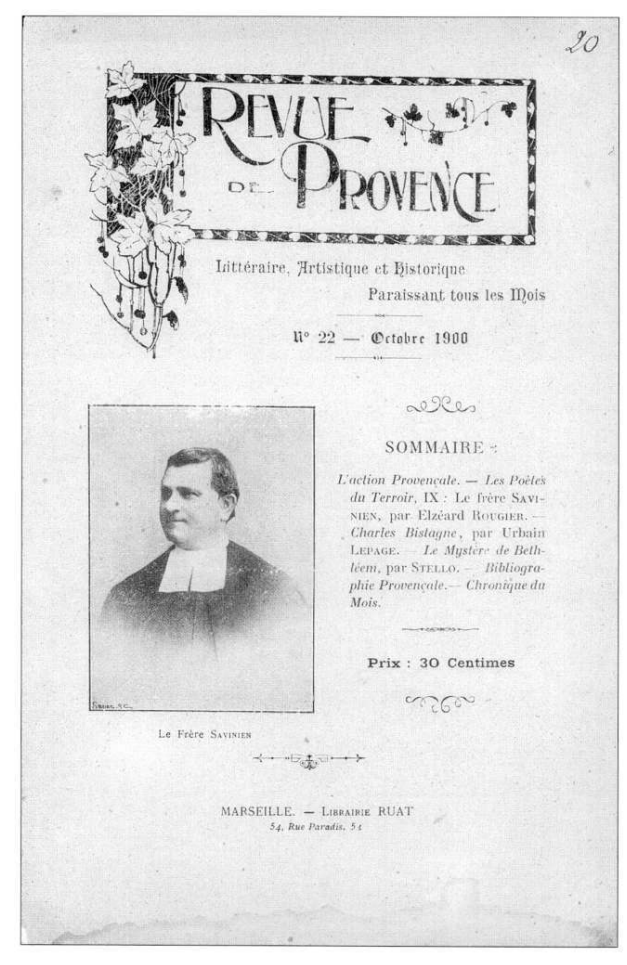
primaire, on présentera la méthode des versions provençales et son histoire depuis 1875 jusqu'au début du $\mathrm{XX}^{\mathrm{e}}$ siècle; on s'interrogera pour finir sur les conditions de la réussite ou de l'échec de la volonté de changement en matière d'enseignement ${ }^{2}$. 


\section{Problèmes linguistiques de l'école primaire au XIX siècle}

2 De l'époque de la monarchie de Juillet, avec en particulier la loi Guizot de 1833, à la Troisième République, avec les lois Ferry au début des années 1880, et au-delà, tous ceux qui cherchent à aboutir à un enseignement primaire ouvert à tous les enfants du pays se trouvent confrontés à un problème sérieux : une majorité d'élèves rencontre la langue française pour la première fois en arrivant à l'école. Cela est particulièrement le cas bien entendu à la campagne, dans un pays où la population urbaine ${ }^{3}$ ne commencera à être majoritaire que dans les années 1930. Or la principale aire linguistique non francophone est celle des "parlers méridionaux» de langue d'oc (le terme "occitan» ne s'impose qu'au cours du XXe siècle), qui couvre un tiers de la France. Certes la régression de l'usage de «l'idiome local ", identifié communément par le terme très négatif de "patois », est une donnée clairement identifiée depuis au moins le XVI ${ }^{e}$ siècle. Mais si, après la Révolution, la bourgeoisie méridionale ne parle plus en langue d'oc, il n'en est pas de même pour le peuple .

3 Le mouvement du Félibrige se trouve dès lors confronté à une contradiction : défendre une renaissance de la langue d'oc, tout en faisant face à l'accusation récurrente de séparatisme. C'est en effet un moment de l'histoire de l'Europe où l'éveil des nationalités a tôt fait d'amalgamer langue et État, comme le montre de façon exemplaire le développement sur tout le siècle de l'unité allemande. Or c'est l'Allemagne qui remporte la guerre de 1870, et d'ailleurs annexe les départements germanophones de l'Alsace et de la Lorraine, tout comme la France avait rattaché à son territoire Savoie et comté de Nice dix ans plus tôt, pour des motifs linguistiques identiques 5 . Après la " débâcle ", tout ce qui menace l'unité nationale est suspect, d'autant plus d'ailleurs que la guerre civile de 1871 provoque d'autres raisons de méfiance entre bourgeoisie au pouvoir et peuple des villes. Dans ces conditions, la République étant, selon le mot fameux de Thiers, «ce qui nous divise le moins", il devient impérieux pour la bourgeoisie républicaine de prendre en main l'appareil scolaire, en vue d'unifier les futurs citoyens sur ses valeurs et sa langue.

4 Pour ce qui est des valeurs, le conflit est ouvert évidemment avec l'Église, longtemps seule institution enseignante, et alors fort peu encline aux perspectives républicaines, Église dont le fleuron scolaire est représenté par les frères des Écoles chrétiennes. La congrégation fondée à la fin du XVII ${ }^{e}$ siècle est la plus importante en France. Sa règle fort en avance sur son temps, est fondée sur le principe de plusieurs maitres dans la même école, qui se répartissent les élèves en fonction de leur niveau scolaire, et dispensent un enseignement gratuit : toutes conditions qui faisaient que les frères n'étaient présents que dans des communes d'une certaine importance.

5 Depuis des siècles que le problème se pose, l'État sous ses différentes formes a évité des mesures brutales pour imposer la langue nationale, ce qui ne veut pas dire qu'il y avait indifférence de sa part. Les autorités ont en général compté plus sur le prestige de la langue du Pouvoir et du Progrès et sur le temps, que sur la coercition. On peut justement en juger dans le domaine de l'Instruction publique en constatant que le règlement modèle des écoles primaires après la première grande loi de 1833 se garde bien d'indiquer quelle langue est utilisée à l'école. 
6 Il faut attendre l'après - loi Falloux de 1850 pour voir apparaître un article : «Le français sera seul en usage dans l'école», d'ailleurs modulé dans un bon tiers des règlements départementaux. Le modèle de règlement du temps de Ferry se contentera de reprendre la formulation, mais les adaptations départementales deviennent alors l'exception.

Face à la persistance de l'usage des « idiomes locaux », l'Église ne rechigne pas, pour sa part, à laisser utiliser les vernaculaires pour prêcher et enseigner au moins oralement le catéchisme, ce qui lui vaut souvent les reproches des instituteurs chargés de le faire réciter, au moins jusqu'à la loi de laïcisation de Jules Ferry.

7 L'autorité centrale de l'Instruction publique n'engage donc pas de mesures générales coercitives, et laisse les maîtres faire face aux problèmes. L'attitude consistant à ne pas tenir compte de la situation, autrement dit à faire comme si les élèves, en particulier les plus jeunes, comprenaient ce qu'on leur disait, n'était guère tenable. Pour autant, en règle générale, les maîtres n'utilisaient guère ce que leurs élèves possédaient comme équipement linguistique initial, préférant considérer qu'ils avaient tout à apprendre.

Il revint au linguiste Michel Bréal de faire entendre une position différente, à partir de son expérience savante. La nouvelle linguistique et la dialectologie naissante faisaient valoir en effet l'intérêt des " patois ", au moins comme témoins privilégiés de langues qui n'avaient pas eu la chance historique d'être devenues langue de l'État. D'autre part l'engouement pour le folklore et la littérature populaire était à la mode dans toute l'Europe lettrée, à la suite de la vague romantique. Et comme il avait des origines alsaciennes, une excellente connaissance de la science et de l'éducation allemandes alors admirées de toute l'Europe, un prestige de savant devenu professeur au Collège de France à trente-deux ans, des convictions républicaines volontiers anticléricales mais nettement conservatrices, ce que disait Bréal eut un écho certain jusqu'au sommet de l'État. Il affirmait donc que les patois étaient non pas des adversaires mais des auxiliaires précieux pour apprendre la langue nationale, dès lors que, comme c'était le cas le plus souvent, les maitres pouvaient s'appuyer sur leur connaissance pour pratiquer des comparaisons éclairantes en vue d'apprendre le français. Ce plaidoyer était contenu dans un livre écrit pendant l'année terrible de 1871 et qui marquera tous les réformateurs des années à venir: Quelques mots sur l'instruction publique en France - le titre était déjà un hommage à Locke - s'appuyait sur la tradition des Lumières, notamment sur Condillac, le fameux précepteur du prince de Parme qui préconisait d'aller « du connu à l'inconnu » par la méthode « intuitive », donc de partir de l'élève, tout comme Jean-Jacques Rousseau. Principes d'ailleurs bien plus utilisés en Allemagne, faisait remarquer Bréal, à la suite de l'occupation de 1809 par la France : si les Allemands avaient pu devenir plus forts que les Français à la suite d'une défaite, en utilisant en quelque sorte le génie des vainqueurs, alors il fallait pour le moins maintenant faire comme eux. Bien entendu, les successeurs républicains d'un Empire terminé en catastrophe avaient confiance dans leur idéologie pour surmonter les risques du suffrage universel (au moins masculin) : voilà pourquoi ils préconisaient une instruction répandue auprès du plus grand nombre, à la différence de ceux qui avaient peur des « classes laborieuses » au point de leur rationner l'instruction.

Mais entre enseignement primaire et enseignement secondaire, si l'idéologie pouvait être similaire, les différences de public restaient totales. Les traditions de l'enseignement de la langue nationale aussi : le secondaire restait marqué par l'apprentissage du français à partir du latin. C'est ce modèle de prestige que le frère Savinian va utiliser pour les enfants du peuple. 


\section{Un projet innovateur... qui suscite des résistances} et félibre. Précédé par un ensemble de travaux érudits qui soulignent l'importance et le prestige de la langue et de la littérature d'oc avant la croisade des Albigeois du XIII ${ }^{\mathrm{e}}$ siècle, le mouvement de renaissance impulsé par Mistral et les poètes du Félibrige à partir des années 1850 , exalte la langue populaire du Midi, encore très vivante malgré des siècles de mise à l'écart, que la désignation par le terme de patois symbolise parfaitement. Mais devant la généralisation de la scolarisation en français, le Félibrige, et Mistral notamment, deviennent vite conscients de l'importance de donner à la langue d'oc une place à l'école. Les difficultés sont évidentes pour une langue qui n'a pas eu la possibilité d'être une langue d'État moderne, donc de pouvoir, ni de bénéficier d'une réflexion étendue pendant des siècles sur sa forme écrite et sur les conditions de son enseignement. Ses détracteurs ont beau jeu de faire valoir les inconvénients de ses variations géographiques, au point que toute comparaison avec le français est accablante; d'autant plus facilement que pour ce dernier et à l'inverse, sont soigneusement dissimulées toutes ses dimensions variationnistes. Mais dès lors comment faire, dans un contexte où le français est si étroitement lié à l'unité nationale, mise à mal par une annexion à la suite d'une défaite, et une guerre civile réprimée sévèrement?

11 Savinian va tout simplement appliquer pour les enfants du peuple la méthode mise en œuvre dans le secondaire pour les enfants « bien nés » : ceux-ci apprennent le français en traduisant du latin, ceux-là traduiront du provençal en français. Un « prospectus » de 1875 montre que son projet est déjà assez avancé à cette époque. La description que Savinian donne de sa méthode est en effet très simple :

«Vous pourrez apprécier vous-même ce travail, par la traduction d'un poème de Mistral : « les Saintes `, que vous trouverez ci-joint.

» Les élèves qui s'inspireront à une source si belle et si pure acquerront des avantages précieux qu'il leur était impossible d'obtenir en étudiant la grammaire ou le style selon la méthode des pensionnats et des cours professionnels.

»Celle que j'ai essayée, consiste à faire une version immédiate, sans être obligé de lire en provençal ; après l'explication d'une strophe de huit vers ou d'un fragment de prose ayant à peu près la même étendue, l'élève traduit à la maison ce devoir journalier et le lendemain le professeur corrige les deux ou trois premières copies ainsi que les deux ou trois dernières, puis il fait écrire au net et par toute la classe, la traduction insérée à la fin du livre du maître. ${ }^{6}$

12 Toutes propositions banales, si l'on excepte le support initial ${ }^{7}$; on remarque bien que les élèves visés sont les plus âgés, ceux, en petit nombre, qui prolongent leur séjour à l'école, d'où la référence à la « méthode des pensionnats et des cours professionnels ».

Le Recueil de versions pour l'enseignement du français en Provence par un professeur. Troisième partie paraît en $1876 \mathrm{chez}$ « Aubanel frères, éditeurs imprimeurs de N.S.P. le Pape et de Monseigneur l'Archevêque ", avec une version pour l'élève et une pour le maître, soit l'addition dans ce cas de la traduction des morceaux présentés. La préface ne manque pas de se réclamer de Bréal, que Savinian connaît par l'intermédiaire de Mistral, sans doute au moment du congrès de la Société des langues romanes tenu à Montpellier en 1875, dont Bréal était le vice-président ${ }^{8}$. La référence à ce dernier sera désormais constante chez Savinian; il commence par une citation très adéquate : "Un savant professeur du collège de France a dit : ‘ Loin de nuire à l'étude du français, le provençal ${ }^{9}$ en est le plus utile 
auxiliaire. On ne connait bien une langue que quand on la rapproche d'une autre de même origine. > - (Michel BRÉAL : Quelques mots sur l'instruction primaire en France. Paris, librairie Hachette) ».

Savinian prévient aussitôt la polémique latente contre ceux qui défendent la langue d'oc: il ne s'agit pas de l'enseigner en concurrence du français. C'était reprendre aussi les principes de Bréal. Savinian en profite pour régler la question des variations du provençal, objection classique, au moyen de l'affirmation de la supériorité intrinsèque du dialecte rhodanien sur tous les autres, ce qui était bien sûr justifié par Mistral, mais linguistiquement discutable, compte tenu en particulier de la graphie choisie.

"Mais tout en admettant l'efficacité de la traduction et l'excellence de la littérature provençale, on supposera peut-être que ce procédé n'aura d'application que dans quelques localités restreintes. Il n'en sera point ainsi.

»Les enfants de toutes les provinces du Midi n'éprouveraient aucune difficulté sérieuse à suivre cette méthode: les essais que l'on a tentés le prouvent victorieusement. En effet, on ne veut pas apprendre le provençal aux élèves. Pour eux c'est la langue maternelle, c'est la langue du cœur, et ils en ont la pleine possession; la diversité de l'orthographe et de la prononciation ne constituent pas un obstacle réel, puisqu'il ne s'agit ni d'écrire ni de parler un idiome.

" L'élève fait directement la version française, et les dialectes n'ont pas assez de différence pour que, dans des pays méridionaux très-éloignés, cette version ne soit pas la même.

»[...] Le provençal, ainsi que le grec et l'italien, se divise en plusieurs dialectes : le limousin, le gascon, le languedocien, le marseillais et le rhodanien; ce dernier est le plus riche : nous y avons puisé largement pour la composition de ce livre. »

Enfin, et pour répondre à l'accusation de séparatisme, il ne fallait pas moins d'une belle citation patriotique de Mistral lui-même montrant l'attachement de la Provence à la France, avant de conclure: "Aux accents si doux de la poésie provençale, le jeune étudiant sentira son cœur tressaillir, son intelligence s'éveiller, et naitre en lui le généreux désir de traduire en bon français les beautés du provençal. $\aleph^{10} \mathrm{O}$ aura remarqué que Savinian avait d'abord publié la Troisième partie de son Recueil. Au dos de l'ouvrage figurait l'ensemble prévu :

«En préparation: Première et deuxième partie du Cours» Ouvrages qui complèteront cette nouvelle méthode d'enseignement du français

»1) Traduction en provençal de Télémaque et fragments des plus beaux poèmes épiques de la littérature universelle

"2) Epopée provençale.»

16 Les trois parties correspondent sans doute aux trois niveaux classiques d'alors de l'enseignement primaire soit, de la première à la troisième, cours élémentaire, moyen, supérieur, devenus un peu plus tard préparatoire, élémentaire, supérieur ${ }^{11}$. Savinian publiera bien après la première partie, celle qui concerne le niveau d'entrée à l'école, assurément la plus difficile. Quant aux ouvrages de complément, l'épopée provençale annoncée sera LaLionide, publiée bien plus tard encore en 1911. La traduction du Télémaque semble avoir été remplacée par un autre ouvrage, Fermin et Testedor (Firmin et Tête d'or), plus adapté aux enfants de milieu populaire, mais qui restera finalement inédit. Il est vrai que dès les années 1850, l'usage de Télémaque comme livre de lecture, très répandu encore jusque dans les années 1830 dans les écoles populaires, est considéré comme suranné, et il n'est plus en usage dans les années 1870 que dans le secondaire...

"Les essais que l'on a tentés le prouvent... », faisait remarquer l'auteur : la mise en œuvre de la méthode au niveau de sa Troisième partie, même si c'est donc avec les élèves les plus 
âgés et les moins nombreux, est un argument évidemment fort. Quelques détails supplémentaires sont fournis dans la préface à la Deuxième partie publiée en 1878 :

«La troisième partie du Recueil des versions provençales a été mise à l'essai dans quelques classes pendant deux ans et la réussite a

démontré que, de Nice à Bayonne et de Perpignan à Limoges, on pourrait obtenir de notables progrès par cette nouvelle méthode.

» Plusieurs inspecteurs primaires l'ont recommandée à leurs instituteurs. »

18 Al'évidence Savinian a utilisé le réseau méridional des écoles des frères, et même peut faire état d'un franchissement de la cloison avec l'enseignement public laïque, même si «plusieurs inspecteurs primaires» est une formule qui n'est pas sans ambiguïté. On trouve d'autres informations dans le plaidoyer présenté devant la hiérarchie de la congrégation en $1884^{12}$. Savinian y reproduit deux textes d'élèves, l'un n'ayant pas suivi la méthode des versions, l'autre l'ayant pratiquée. La supériorité du dernier est plus qu'évidente ; il faut dire pourtant que cette tentative de preuve expérimentale paraît fort peu convaincante, tant sont nombreuses les critiques qu'on peut en faire, à commencer par son caractère unique.

19 Mais revenons sur cette Deuxième partie publiée en 1878. Alors que la Troisième partie initiale était signée anonymement par « un professeur », celle-ci l'est par une "Société littéraire ", ce qui peut signifier la dimension non plus individuelle mais collective, du Félibrige lui-même, ou des frères, mais on verra qu'il n'en est rient ${ }^{13}$. Dans une préface étendue, sont développés les avantages de la traduction dans trois directions: pour l'orthographe, pour le style, pour la morale patriotique, avec citations de Bréal à l'appui ${ }^{14}$. Nous sommes ici un peu en retrait des débats d'actualité : savoir si la discipline naissante $\mathrm{du}$ « français » pour les enfants du peuple devait être configurée vers la dictée ou vers la composition française ${ }^{15}$.

Les textes de cette Deuxième partie sont plus courts, parfois non signés, allant jusqu'à des listes de proverbes ${ }^{16}$, avec un lexique associé à chaque texte: appareil pédagogique minimal, mais qui correspond bien à des élèves plus jeunes. Bréal figure dans la préface au titre des "témoignages d'approbation", avec cette citation, qui a l'air cependant d'être tirée d'une correspondance de courtoisie : «Vous verrez que cette méthode sera suivie jusque sur les bords de la Loire."

21 On peut donc penser que la congrégation, sans pour autant embrasser ses opinions, laisse à Savinian l'opportunité de développer son innovation, tout comme son usage initial avait donné lieu à l'accord du Supérieur de la congrégation ${ }^{17}$. Ainsi y a-t-il des "versions flamandes et provençales" présentées à l'Exposition universelle de $1878^{18}$. De fait les résistances internes existent, comme le prouve le contenu d'un article de Savinian, publié par le Messager de Toulouse en $1881^{19}$, avec des arguments que l'on pourrait trouver chez bien des innovateurs en pédagogie :

«N'allez pas croire pourtant que les congréganistes aient tressé des couronnes à leur confrère, créateur de ce système plein d'attrait et d'un succès bien constaté. Mon Dieu, non!

»Rien n'est plus long et ne rencontre plus d'obstacle que la marche progressive de la pédagogie. C'est une souveraine un peu routinière, solidement établie sur un trône séculaire. L'Universitée $\left.{ }^{2}\right]$ les institutions congréganistes la soutiennent à la fois, et, empressées dans les hommages qu'elles lui rendent, elles forment une barrière que le progrès ne peut pas toujours franchir. Voilà ce qui est arrivé, mais l'Université comme les congrégations, toutes agissent avec une pure bonne foi, et nous espérons que, se rendant mieux compte des services que la découverte à 
laquelle nous faisons allusion doit rendre à l'enseignement, elles l'appelleront en aide au français qui végète à l'école primaire. " officielle des «idiomes locaux », l'impatience grandit et l'on trouve bientôt dans les rangs des félibres une revendication plus radicale : donner un statut scolaire à part entière à la langue d'oc.

Invité en 1890 à faire le discours de Sceaux - où les félibres parisiens tiennent leur fête annuelle en invitant une personnalité notoire - Bréal, après avoir rappelé ses positions sur l'intérêt des « patois » en général, du provençal en particulier, va s'en prendre sans le nommer au procédé de Savinian.

«J'applaudirai tout à l'heure les lauréats de vos concours de poésie. Mais je ne crois pas que le dialecte doive faire partie du programme officiel de l'école. Il y a quelques années, un félibre, d'ailleurs bien intentionné, a proposé, pour les écoles $\mathrm{du}$ Midi, des thèmes provençaux et des versions provençales. Faut-il appliquer au parler natif les méthodes savantes qui nous permettent à grand-peine de retenir quelques mots de latin et de grec! Je ne le pense pas. A ceux qui savent le dialecte, ces exercices paraitraient trop faciles, et ils n'apprendraient pas grand-chose à ceux qui ne le savent pas. Il faut désirer que l'idiome paternel ne rappelle à nos enfants que des souvenirs sans mélange. Mais ce que nous avons le droit de demander, c'est que l'instituteur ait la considération qui convient pour un langage français, et qui, bien qu'il ne soit pas le langage officiel, n'en a pas moins ses lois régulières : si le maître est bien inspiré, il le fera intervenir de temps en temps pour éclairer un mot, pour montrer une parenté, pour laisser entrevoir une origine. Il n'en faut pas plus : 
on dissipera ainsi les préventions et l'on rectifiera les idées fausses. C'est le plus sûr moyen de faire respecter et aimer nos vieux idiomes provinciaux. $»^{24}$ ses conférences aux étudiants de Sorbonne, après s'être adressé aux professeurs de langues vivantes, ouvrant avec eux un lourd contentieux : l'enseignement d'une langue vivante ne doit pas utiliser le modèle classique de l'enseignement du latin, langue morte, enseignement qui n'a pour ambition que d'enrichir l'esprit, alors que l'objectif de l'enseignement d'une langue vivante est de la parler, d'où la promotion qu'il veut assurer à la "méthode directe ", à la suite de l'Allemand Viëtor. De plus, et quelle que soit sa sympathie pour le Félibrige et Mistral, Bréal se méfie visiblement du risque de donner une place à l'école à une autre langue que le français. Les polémiques qui ont fait suite à la Sainte Estelle d'Avignon de $\left.1888{ }^{(25}\right)$, faisant à nouveau renaître l'accusation de séparatisme, ne sont sans doute pas étrangères à ces précautions.

Savinian va répondre dans un long plaidoyer, qui parait certes dans deux journaux parisiens, mais il s'agit de feuilles ultracatholiques au tirage confidentiel ${ }^{26}$.

Savinian commence par citer Charles Maurras (le futur nationaliste de l'Action française n'est alors qu'un jeune félibre venu faire du journalisme à Paris) : «M. Bréal s'est donné, à mon sens, le tort de combattre ce système d'éducation : a priori, il parait excellent, et, en fait, il n'a formé jusqu'ici que d'excellents sujets ${ }^{27}$.

L'opposant à Bréal, Savinian en vient à présenter son argumentation, où perce vite l'amertume :

«Voilà un choc d'opinion assez éclatant; que la lumière en jaillisse! Les écoles méridionales et l'enseignement du français y ont un intérêt majeur. N'oublions pas que des milliers d'instituteurs hésitants nous écoutent; et, selon l'impulsion reçue, le progrès scolaire, véritable Protée, nous échapperait encore ; ou nous réussirions [sic] à le fixer dans nos écoles.

»Cela montre bien qu'avant de se prononcer définitivement, il faut de longs débats précédés d'études approfondies, soutenus par l'expérience quotidienne, appuyés sur des résultats écrits ou oraux, apportant la sanction des savants et des praticiens d'une compétence décisive. [...]

» Eh bien! M. Bréal, malgré son érudition, son esprit méthodique, n'a pas, croyonsnous, réuni tous les éléments résolutoires exigés par le rejet ou l'admission de la méthode des versions provençales françaises. Il est presque le seul qui en ait traité publiquement dans ce sens [...].

» [...] Depuis bientôt quinze ans, M. Bréal connaît cette méthode, il a eu mainte occasion de se prononcer contre son introduction dans l'école, jamais, que nous sachions, il n'a fait entendre un mot contradictoire dans la presse ou dans des conférences; et c'est après cette longue période d'une élaboration locale dont il resta toujours éloigné, sans pouvoir ainsi être initié aux avantages de la découverte, qu'il en déclare l'inutilité. Mais il se heurte à l'adhésion d'un directeur d'école du département des Landes qui écrit à l'auteur des versions: « Je vais composer les mêmes livres pour le dialecte gascon ,, à celle d'un rédacteur du journal pédagogique de Nîmes, disant : ‘avec votre système, vous avez pleinement raison ', à l'approbation du jury de Londres qui accorda un diplôme d'honneur en participation, portant la mention très bien, à l'exposition scolaire de Digne qui décerna une médaille d'or.

» Tout cela est bien fait pour laisser à M. Bréal des doutes sur la sévérité de son exécution, et nous n'appelons pas en témoignage tous les partisans de la traduction classique; ils sont nombreux, et ils ne manquent point de compétence, dans l'enseignement secondaire, soit celui de l'Université, soit celui des écoles libres. Au fond, de quoi s'agit-il? De traduire les chefs-d'œuvre d'une langue pour mieux apprendre le français; au collège, c'est la méthode des enfants du riche, à l'école 
primaire, ce sera celle des enfants de nos classes laborieuses.

»Par ce temps de démocratie et d'égalité raisonnable, procéder différemment serait chose fâcheuse.

» Ou la traduction est bonne ou elle ne vaut rien; si la méthode est fausse, d'où vient que vous la maintenez avec les collégiens; si vous la jugez réellement utile, pourquoi la proscrire dans les classes des familles populaires?

[...]

»M. Bréal est entré dans une voie de progrès et nous l'en louons volontiers; il mérite par là les remercîments enthousiastes que les félibres de Sceaux ne lui ont pas ménagés au nom de l'éducation méridionale, mais pourquoi s'arrêter à l'éclaircissement d'un mot français par la terminologie en langue d'Oc ? [...] » soutiens, dont il n'a pas l'air d'avoir conscience. Il revient ensuite sur les avantages de la version pour le «style ", c'est-à-dire l'apprentissage de la production écrite, comme on dit aujourd'hui, permettant notamment la correction simultanée pour tous les élèves d'une classe.

Il reprend l'argumentation "contre la méthode des langues mortes": comme le provençal est connu, il n'a pas besoin d'études préparatoires. Enfin, et on peut s'étonner de la faiblesse encore de cette partie finale, il explique les réticences " en haut lieu » par la méconnaissance des « ressources du Midi », avant de citer Jules Simon ${ }^{28}$ évoquant lors de la fête de Sceaux de 1889 la perspective de Mistral occupant une chaire de provençal à l'Université pour l'aider à obtenir un fauteuil à l'Académie. Et Savinian de regretter que Bréal n'ait pas revendiqué au même endroit une place pour le même Mistral au Conseil supérieur de l'Instruction publique ${ }^{29} \ldots$

31 Savinian, on l'aura remarqué, s'efforce de s'appuyer sur des reprises de sa méthode hors du milieu plus restreint qui lui est propre, et qui dispose d'un prestige porté par les investissements de l'État, l'enseignement public. Il s'agit de convaincre les maîtres et leur hiérarchie: entreprise rendue difficile compte tenu de sa situation dans l'autre camp. C'est alors qu'il joue de son autre appartenance, celle du Félibrige. Ainsi il prend luimême des initiatives, en s'adressant à deux jeunes instituteurs, d'abord Antonin Perbosc, dont il a peut-être entendu dire qu'il avait fait ouvrir dans un journal pédagogique national, La Tribune des instituteurs, un débat de plusieurs mois autour des patois à l'école, débat conclu sur l'intérêt de leur usage pour apprendre le français ${ }^{30}$. Une lettre insérée dans le recueil LE 5792 (p. 79) vient confirmer aussi qu'il s'était adressé à un autre instituteur, ami de Perbosc, Prosper Estieu. Les occitanistes les connaissent comme poètes d'importance, mais aussi comme militants d'une nouvelle graphie ayant l'ambition d'unifier le champ occitan. Le début de cette lettre, ci-dessous reproduit, fournit un témoignage sur l'état de l'opinion des instituteurs, qui n'infirme pas l'analyse faite en $1878^{31}$, et le prestige dont Savinian commence à jouir au moins dans la mouvance du Félibrige, y compris auprès de gens de gauche comme Perbosc ou Xavier de Ricard. Mais le projet d'Estieu d'adapter pour le languedocien la méthode savinienne n'aura pas de suite exacte.

«Fraïssé-Cabardès, par Cuxac-Cabardès (Aude) Le 12 janvier 1893 Mon cher confrère,

» c'est avec le plus grand plaisir que j'ai lu la charmante lettre que vous venez de m'adresser. Vous n'étiez pas un inconnu pour moi. Plusieurs fois, j'ai eu l'occasion de parler de vous avec mes amis de Ricard et Antonin Perbosc, notamment en septembre dernier à Toulouse. Oui, il faut vaincre à tout prix. Mais que d'hostilités et d'indifférences n'avons-nous pas à surmonter! Les hostiles, vous les connaissez autant et plus que moi ; mais ne pensez-vous pas que les indifférents ou les timorés 
sont surtout les instituteurs, pris en bloc? Saturés des doctrines universitaires et unitaristes, ils ne pensent guère par eux-mêmes ; de plus, ils sont sous le joug, et, eussent-ils une volonté bien arrêtée, ils se garderaient bien de la manifester - dans ma région, surtout. C'est parce que je suis on ne peut plus féru de l'enseignement de la langue française par la méthode comparative que j'assombris le tableau. Néanmoins, tout cela ne doit pas nous refroidir. Comme vous, je voudrais, de concert avec mon ami Perbosc, publier un système complet d'enseignement de la langue pour nos écoles et nos familles haut-languedociennes. Nous ne pourrions le faire qu'en nous inspirant - et nous le proclamerions - de vos travaux que nous voudrions bien connaître en totalité. Si cela vous est possible, veuillez donc me les communiquer et, aidé de mon ami, un vaillant et un loyal doublé d'un grand artiste, nous tâcherons de les adapter à notre façon à notre dialecte qui, bien étudié, se rapproche du vôtre, c'est à dire celui de Mistral, plus que ne le pensent nos détracteurs. [...]»

Six ans plus tard, une nouvelle polémique rebondit, mais cette fois l'affrontement est plus direct. En 1896 donc, les amis de Savinian, en particulier montpelliérains, arrivent à le faire intervenir à Paris au congrès annuel des Sociétés savantes, obtenant un certain écho dans la presse catholique et de droite. Le congrès d'Avignon qui lui fait suite rassemble pour la première fois semble-t-il des partisans de la langue d'oc, du breton et du basque, en vue de promouvoir ces langues à l'école, à la chaire et à la tribune. La polémique rebondit avec les journaux républicains. L'Éclair, que nous avons déjà rencontré, un journal de droite nationaliste, obtient une interview de Bréal, qui cette fois s'en prend nommément au frère Savinian, et rappelle les limites classiques : pas question de donner au provençal une place comme langue apprise ${ }^{32}$.

« Je connais le frère Savinien depuis longtemps. C'est un homme intéressant, rempli d'ardeur et de zèle, mais on me permettra de douter de l'efficacité de sa méthode, et surtout d'en contester la justesse et la vérité.

» Ce serait tout bonnement reléguer le français au rang des langues mortes, c'est-àdire enseigner notre langue nationale de la même façon qu'on enseigne au lycée le grec et le latin. Et cela au moment précis où la précieuse méthode des langues parlées l'emporte sur le système indigeste des thèmes et des gros dictionnaires!

« Remarquez bien que je n'éprouve aucun sentiment d'hostilité envers les dialectes locaux, car j'aime le provençal et je me délecte de la lecture savoureuse de l'Aiòli, qui s'imprime dans le Midi ; mais j'estime que le provençal, aussi bien que les autres "parlers », doit rester ce qu'il est: le langage de la famille et du foyer. Trois ou quatre grands pays [sic : poètes ?] du Midi l'ont épuré, affiné, amenuisé, si j'ose dire, en sorte qu'il apparaît un peu factice par endroits. La langue de Mistral décèle parfois ce côté artificiel, malgré sa richesse, sa souplesse et sa suave harmonie. Et j'imagine que le poète de Maillane, qui possède, en plus de la poésie, de hautes qualités intellectuelles, n'approuve guère en son for intérieur cette méthode d'enseignement. Mais, comme il est chef, il se voit obligé de suivre ses disciples...

" Le provençal de la rue, de la Canebière et du vieux port diffère beaucoup du provençal que chantent Mistral, Aubanel et Roumanille. Il diffère sensiblement de celui que Savinien enseigne avec ses grammaires provençales; c'est dire que les élèves sont forcés d'apprendre simultanément le parler local et la langue nationale. Et c'est peut-être bien l'idée secrète de certains félibres, continue M. Bréal en souriant finement; ces enfants terribles voudraient substituer peu à peu le provençal au français. Or je vous demande ce qu'il adviendra du langage philosophique ou sociologique, par exemple, interprété en langue d'Oc! Non, voyez-vous, on apprend aujourd'hui l'anglais en causant anglais, l'allemand en causant allemand, et l'on n'apprendra bien le français que par la parole française, la parole vivante. " 
craintes anciennes à l'égard des tenants des idiomes régionaux s'étaient réveillées avec le manifeste des jeunes félibres parisiens de 1892, qui cherchaient à sortir de la routine de leurs aînés en faisant deux propositions politiques que Mistral avait jusque là soigneusement évitées : "C'est nous, jeunes félibres, qui demandons l'autonomie provinciale et l'enseignement de la langue d'oc dans les écoles du midi. Nous n'avons jamais songé à la faire prédominer sur le français, nous réclamons seulement son droit de cité, car actuellement il est enjoint aux instituteurs de défendre aux enfants de parler languedocien. ${ }^{33}$

Le conflit interne au Félibrige, dont Bréal a visiblement connaissance, n'épargne pas Savinian, accusé de minimalisme, pour ne pas revendiquer un enseignement du provençal à l'école : le témoignage de Frédéric Mistral neveu est sur ce point éclairant ${ }^{34}$. Le dossier EL 5792 contient à la fin de son premier tome (p. 186) un texte manuscrit de Savinian reproduit ci-dessous, et qui s'en tient soigneusement à répondre à Bréal. A notre connaissance, cette réponse ne fut jamais publiée. Son ton plutôt vif peut l'expliquer.

« Réponse À l'article de l'Éclair

»1) M. Bréal se trompe en affirmant [« insinuant» au dessus] que ces enfants terribles - les félibres - voudraient peu à peu substituer le provençal au français.

»Cela n'existe que dans son imagination.

"2) (Ce serait tout bonnement reléguer le français au rang des langues mortes, c'est-à-dire enseigner notre langue nationale de la même façon qu'on enseigne au lycée le grec et le latin. >

« Langue morte le français!

« Mais pas du tout : parce que sur un point la méthode ressemble à celle du lycée connaissant le latin ou la langue d'oc on s'en sert pour mieux apprendre le français - il ne s'ensuit pas que le français soit considéré comme une langue morte. Dès que ce travail de version est fait au lycée on abandonne le latin; et lorsque la langue d'oc a rendu ce service, on l'abandonne aussi à l'école primaire pour reprendre l'enseignement exclusif de la langue française écrite ou parlée, mais toujours vivante

«Le sophisme de M. Bréal vient de ce qu'il s'est dit : L'enfant au lycée étudie le latin langue morte par la langue française et il étudie en primaire le français par la langue maternelle donc le français est enseigné là comme une langue morte. « $\mathrm{M}$. Bréal s'est mépris.

» [verso] Car alors il y aurait les mêmes conditions pour les études de l'anglais, de l'italien, de l'allemand puisqu'elles sont étudiées comme le latin, avec thèmes et versions [ces 4 mots au dessus], et selon M. Bréal cela suffirait pour dire qu'elles sont reléguées elles aussi au rang de langues mortes!

"3)<J'estime que le provençal doit rester ce qu'il est ; le langage de la famille et du foyer. '

» Où s'est-il aperçu qu'on l'avait rendu le langage de l'école?

" Jusqu'ici on s'en est tenu absolument aux conseils qu'il donnait lui-même pour ‘ la lecture du provençal à l'école, affirmant qu'il y était le plus utile auxiliaire du français [ces 2 mots au dessus]. , »4) «Non voyez-vous, on apprend aujourd'hui l'anglais en causant anglais, l'allemand en causant allemand, et l'on n'apprendra bien le français que par la parole française vivante. )

«Pourquoi M. Bréal, maître puissant dans l'Université a-t-il attendu ce moment pour proclamer un principe qui doit révolutionner l'enseignement classique? L'aima parens ce qui étonne, n'a pas l'air de s'en douter.

« Allons MM. Les professeurs de grec et de latin, fermez vos livres, emportez votre ‘ système indigeste de thèmes et de gros dictionnaires. > on n'apprend pas au lycée le latin et le grec pour écrire, pour parler ces langues, pour les savourer; on ne s'en sert pas non plus pour mieux apprendre le français. À quoi bon tant de dépenses de temps et d'argent?

» Et ces Messieurs sur la parole du Maître devront s'en aller afin de ne pas donner 
plus longtemps un exemple qui deviendrait funeste à l'école primaire !

»C'est bien cela que voudrait M. Bréal ; c'est du moins les conséquences forcées de sa doctrine.

» Peut-on bien maintenir le latin au lycée et repousser le latin des pauvres à l'Ecole primaire des enfants du peuple? Qu'en pense M. Bréal. Ah! nous en resterions avec la langue unique méthode qu'il a reconnue inférieure à celle des études comparatives quand il a dit avec raison : ‘ On ne connait bien une langue que quand on la rapproche d'une autre de même origine... > Le patois là où il existe fournit ce terme de comparaison... »

"Ce qu'il y a de plus facheux [sic] dans ces subtiles incohérences et ces flagrantes contradictions, c'est que le mouvement de progrès scolaire risque d'être enrayé, le progrès social, lui aussi. Après tout, pauvre peuple, va! Ils sont eux les riches en science, en littérature, si tu souffres dans les écoles primaires, si tu restes incapable en travaillant de gagner tout ton pain et de réclamer ta part de justice communale et de justice d'état, qu'importe! Avec l'ignorance des masses, les gens diserts, les écrivains, les érudits, les éloquents, les politiques seront toujours assez nombreux pour mener tes affaires ; et ils les mèneront bien! Le français uniquement enseigné ne s'apprend pas aussi bien que par le secours d'une autre langue. Mais là haut dans certaines contrées, ils n'ont pas d'autre moyen; et ils tendent depuis longtemps à t'en dépouiller pour rendre tes études plus médiocres encore.

»Cependant ne crains rien, la pensée est puissante, plus puissante que le métal en fusion qui brise parfois le moule dans lequel il est enfermé. Paris et la Sorbonne comme la France par ses délégués de sociétés savantes [depuis : comme, au dessus] se sont prononcés en faveur de ton éducation, peuple du Midi, travaille espère et tu seras par ta double langue un jour meilleur provençal et meilleur Français. »

L'argumentation de Savinian, si elle ne se renouvelle pas par rapport à sa réponse de 1890, ne manque pas d'habileté pour mettre en évidence les contradictions qu'on pouvait percevoir dans les propos de Bréal, étant donné la situation contrastée entre école primaire des pauvres et secondaire des riches. Dans une certaine mesure, on peut même trouver à Savinian une position socialement plus revendicative, puisqu'il ne manque pas de justifier le développement de l'instruction pour que le peuple se défende mieux, argument si souvent utilisé par les républicains : cependant ceux qui abusent les pauvres à ses yeux sont seulement ceux qui maîtrisent la langue... Quant à sa conclusion, son irréalisme peut surprendre, tant les sociétés savantes, même réunies en Sorbonne, sont loin d'être un lieu de reconnaissance décisif en matière de pédagogie.

En 1902, un nouveau Conseil supérieur des frères des Écoles chrétiennes va enfin adopter la méthode savinienne. Mais en 1903, le président du conseil Combes, le même qui avait promis en 1896 de faire une circulaire pour donner la possibilité d'utiliser dans les classes la méthode des versions provençales ${ }^{35}$, suspend le traitement d'un certain nombre de prêtres bretons et basques qui ne prêchaient pas en français. Et cette même année, les congrégations enseignantes sont interdites. Le frère Savinian réduit à l'état laïque reprend son nom et va tenir une école privée à Bourg-Saint-Andéol puis à Avignon. Savinian publiera en 1911 son épopée provençale, LaLionide, précédée d'une préface de Mistral, et d'une lettre de Maurice Barrès : 550 pages (avec la traduction) pour célébrer la résistance des Provençaux aux Maures...

Cette même année 1911, Jaurès prend position dans le même sens que Bréal, fournissant un soutien de gauche à la préservation de la richesse linguistique de la France.

Bréal est désormais identifié avec Savinian dans la mémoire des partisans des « idiomes locaux ", comme faisant partie de ceux qui ont résisté les premiers à la pression majoritaire de la condamnation, de l'indifférence, ou de l'oubli, même si ce fut sans succès décisif. Le débat de 1925 , suscité par une nouvelle demande des partisans de la 
langue d'oc, et la réponse négative du ministre de l'Instruction publique de Monzie, va susciter l'échange d'arguments déjà anciens, où la méthode savinienne sera toujours présente, après le décès de son créateur.

\section{Pourquoi l'échec}

En février de l'année 1911, Savinien-Joseph Lhermite intervient devant le congrès des jurisconsultes catholiques, dont le thème était « Raviver nos traditions nationales ». Il cite toujours Bréal à propos de l'usage de l'histoire, puis pose les questions classiques d'un innovateur qui se demande pourquoi ce qu'il préconise n'aboutit pas ${ }^{36}$ :

«Les traditions, précieux élément de l'éducation populaire, sont surtout renfermées dans la langue.

«Par l'enseignement du français à l'école primaire, notre système d'instruction publique laisse subsister un phénomène étrange.

«La France est bilingue dans plusieurs de ses provinces.

» On y voit, d'une part, les enfants de la classe sociale supérieure employer le merveilleux instrument de la traduction ; avec cela sont formés les écrivains, les avocats, les docteurs, les savants et les artistes du grand art.

»De l'autre, prohibition absolue de se servir de la traduction, des études de langues comparatives. Et pourtant rien ne saurait les remplacer avantageusement: ni la dictée, ni les études de conjugaison, ni les analyses de grammaire, exercices d'un mode lourd et indigeste, comparés à ceux de la traduction.

«Pourquoi cette différence essentielle de méthode entre les deux ordres d'enseignement?

« Est-ce que l'intelligence des enfants à l'école primaire n'est pas de même nature que celle des autres à l'école secondaire?

"I1 y a là une énorme erreur pédagogique.

" Presque toutes nos plus anciennes provinces ont un dialecte local qui devrait s'unir à l'idiome national pour le meilleur succès des études. Il s'agit d'employer pour le français la langue d'oc que la Revue des Deux-Mondes appelle le latin de l'école primaire. Et l'Occitanie comprend un million d'élèves.

»Cette méthode de traduction leur serait des plus fructueuses; pourquoi les en priver?»

\section{Reprenons l'argumentation :}

- Une grande cause : il y a un problème dans l'efficacité de l'enseignement, par rapport aux objectifs généraux qui font en principe consensus : en l'occurrence, donner à tous les enfants, notamment de milieu populaire, la maîtrise du français, et notamment à ceux qui sont originaires du Midi : « progrès scolaire » et « progrès social » sont associés, comme le dit Savinian dans sa réponse à l'interview de Bréal dans L'Éclair en 1898.

- Des modalités commodes : l'innovation proposée ne présente que des avantages, puisqu'elle s'appuie sur le connu des élèves, ce qui valorise leurs origines, et suscite leur intérêt. Les apprentissages sont plus rapides et plus sûrs, tant avec l'ambition essentielle d'apprendre l'orthographe, que dans le domaine du « style », c'est-à-dire la maîtrise de l'écrit, justement le souci principal des tenants d'une rénovation de l'enseignement du français. L'organisation du travail collectif en classe s'en trouve favorisée. Il existe en plus des manuels pédagogiques utilisables depuis l'entrée jusqu'à la sortie de l'école primaire.

- Un modèle prestigieux : l'innovation s'est faite d'ailleurs sur un modèle valorisé puisque appliqué aux enfants de la classe supérieure. La possibilité de s'appuyer sur la langue d'oc, en pleine renaissance avec une littérature ancienne et nouvelle reconnue par sa qualité, ajoute encore à l'intérêt. 
- Des preuves de réussite : les résultats obtenus dans plusieurs endroits et depuis des dizaines d'années démontrent l'efficacité de la nouvelle méthode.

- Le soutien de grands noms de la science et de la littérature, voire de responsables de l'enseignement public, y compris donc en dehors de la sphère d'influence de l'auteur.

Et pourtant, après des dizaines d'années, il faut constater que la méthode des versions n'a pas emporté l'adhésion d'une majorité, ni même d'une minorité des maîtres.

Voyons maintenant dans l'autre côté de la balance les obstacles rencontrés. Ils sont évidemment sérieux, sans quoi en effet l'on n'aurait pas ce résultat.

- La « grande cause » de l'élargissement et de la qualité de l'enseignement du français doitelle passer par l'entrée officielle à l'école d'idiomes locaux qui peuvent être considérés comme ses concurrents? Les détracteurs de la méthode des versions peuvent le craindre pour des motifs patriotiques, en identifiant l'unité de la nation et l'unité de langue. Certes un rallié prestigieux à la République comme Renan avait montré dans sa fameuse conférence de $1882^{37}$, qu'une nation était non pas fondée par une religion, une race, une langue uniques, mais par la volonté politique de vivre ensemble. Mais cette argumentation était loin d'être unanimement partagée, en particulier par des républicains qui se sentaient les héritiers de l'abbé Grégoire, identifiant le français national à la Révolution, et les patois à l'Ancien Régime, ce qui était pour le moins rapide. Ainsi le célèbre publiciste Francisque Sar-cey, pourtant très sensible à l'argumentaire de Bréal, reste très soupçonneux. À la suite du discours d'Avignon de Mistral (1888) où celui-ci s'inquiétait du recul du prestige de la langue d'oc chez des jeunes filles, un échange a lieu dans le journal parisien Le XIX ${ }^{e}$ siècle. Mistral revendiquait pour les Provençaux d'apprendre le français à partir du provençal comme le disait Bréal, et de ne pas être moins maltraités que les Bédouins à qui l'on consentait l'apprentissage de l'arabe ; Sar-cey prend alors à partie l'auteur de Mirèio ${ }^{38}$ : "Qu'entendez-vous dire par cet apologue; sinon qu'il faut, dans les écoles de Provence, enseigner de préférence la langue du pays et donner la seconde place au français?

"Si ce n'est pas cela que vous avez voulu signifier, c'est cela, je vous assure, que nous avons tous compris: je viens de relire votre discours, c'est cela que je comprends encore.

» Eh bien! mon cher maître, c'est cela que nous n'admettrons jamais, jamais !

» Je ne suis pas exclusif comme Weiss, qui voudrait que l'on proscrivît rigoureusement tout dialecte de l'enseignement primaire. Je l'ai dit dans mon dernier article, et j'ai donné les raisons de cette tolérance. Mais le dialecte ne doit occuper que le second rang dans les préoccupations du maître : il ne doit être pour lui qu'un moyen de mieux enseigner la vraie langue, la langue nationale, le français. "Sur ce point, mon cher Mistral, nous serons intraitables. Ne me parlez pas des Bédouins : les Bédouins ne sont pas Français et n'ont pas envie de l'être. On leur apprend l'arabe en même temps que le français, parce qu'ils sont Arabes et veulent rester Arabes ; parce que tout les sépare de nous, la race, la religion, les mœurs, les institutions, les souvenirs et enfin la conquête. Les Arabes sont un peuple conquis, et ils font bande à part.

" J'aime à croire, mon cher Mistral, que vous êtes, vous et les vôtres, Français et bons Français, de cœur, de race, de mœurs, et de tout. Eh bien! laissez vos enfants apprendre le français!

" On ne forme une même nation qu'à condition de parier une même langue. » Voyez la faute que nous avons commise en Alsace! Au bout de deux siècles, le peuple alsacien ne parlait pas encore notre langue, parce que nous n'avions pas pris soin de la lui enseigner à l'école primaire. Pensez-vous que la résistance qu'il oppose à l'oppresseur n'aurait pas eu un point d'appui plus ferme, si les Allemands n'avaient pas eu pour intermédiaire, avec nos frères séparés la communauté du langage? 
» Chantez-nous de beaux poèmes en provençal : nous les lirons avec ravissement, nous, hommes du Nord, comme nous lisons une page d'Homère, à travers la traduction qui nous aide; mais, pour l'usage ordinaire de la vie, parlons tous le français, puisque nous sommes tous Français. »

- Si l'on en vient aux modalités, l'absence de difficultés n'est pas non plus aussi claire que l'affirme Savinian. L'existence de manuels est avérée pour le provençal, mais il y a nécessité d'outils de travail très différenciés, non seulement pour les régions non-méridionales, mais même pour celles-ci, compte tenu des variations de la langue d'oc forcément sensibles pour une langue surtout parlée et dont la graphie est calquée sur le français ; d'où la nécessité d'avoir des épigones que Savinian trouve d'ailleurs au moins dans les Landes, l'Agenais, les Pyrénées-Orientales... Dans sa thèse, l'abbé Aurouze arrive à moins d'une dizaine de méthodes relevant du « savinianisme » (voir Bibliographie). Il faut donc décliner pour chaque partie du territoire, parfois en dessous même du niveau départemental, des outils pédagogiques, ce qui est loin d'être aussi commode que d'avoir affaire aux éditeurs nationaux, avec leurs capacités de production et de diffusion, leurs revues pédagogiques hebdomadaires, ou, pour une congrégation nationale aussi puissante que les frères des Écoles chrétiennes, qui possède ses collections de manuels, une déclinaison régionale coûteuse. Le contenu chrétien des manuels de Savinian constitue sans doute aussi un obstacle sérieux à leur utilisation directe dans les écoles laïques : « Mais comment [...] introduire dans les classes universitaires des livres imprégnés de l'esprit chrétien, alors que dans les hautes sphères de l'enseignement, le mot d'ordre est de supprimer, dans leurs manuels, le mot même de Dieu! ${ }^{39}$ de 1893, voir supra), et même si l'argument n'est pas rédhibitoire, sauf auprès $\mathrm{d}^{\prime}$ inspecteurs sourcilleux ${ }^{41}$ : «[...] Vous me dites que la réglementation scolaire n'empêche d'expliquer une règle de grammaire au moyen de l'idiome local. Hélas ! voici ce qu'on peut lire dans le règlement des écoles publiques du département de l'Aude : Art. XI, le français seul sera en usage dans la classe. > Et nunc erudi-mini... [...] »

Au-delà des obstacles matériels, le prestige du modèle du secondaire, où le latin permet d'apprendre le français, ne peut pour autant évacuer les débats de l'époque sur la "question du latin ", qui va aboutir à mettre en cause un enseignement fondé sur les seules humanités avec la réforme de 1902. Difficile aussi de faire valoir le prestige de la langue d'oc, qui est loin d'être reconnu dans la " classe supérieure », ou dans le personnel ecclésiastique, comme le montrent parmi bien d'autres les propos de E. Saint-Raymond, se plaignant des " curés franciseurs » et des "évêques parisiennants " ${ }^{42}$.

- Quant aux preuves de réussite, brandies par Savinian et ses soutiens, on a déjà fait valoir que la comparaison entre les compositions françaises de deux élèves n'est guère sérieusement démonstrative, pas plus que le succès aux examens, voire dans un concours de poésie, d'anciens élèves ayant utilisé la méthode savinienne. Il est vrai que l'époque commence à peine à s'intéresser à l'expérimentation pédagogique, en s'inspirant notamment de travaux 
américains avec Alfred Binet. Mais il se trouve que dans un département au moins, le recours à l'usage en classe de la langue locale a été reconnu, sinon un moment encouragé : c'est le cas du basque dans les Basses-Pyrénées, avec des effets induits sur le béarnais, puisque seul l'arrondissement de Mauléon est bascophone. Or le retard constant de la partie basque ne peut plaider en faveur de la prise en compte des perspectives saviniennes; pas plus à vrai dire que celles de la méthode directe, ignorant par principe le vernaculaire, alors portée par l'inspecteur général Carré $\left({ }^{43}\right)$, curieusement absent des préoccupations de Savinian : mais la mise en œuvre en était bien plus simple.

- Le soutien enfin de personnalités de tous bords reste obéré par la tension grandissante dans les années 1880-1910 entre républicains et catholiques : le résultat est que, en dépit de cas d'espèce notables, la référence aux langues régionales à l'école a du mal à s'émanciper de son côté rétrograde. Les responsables du ministère de l'Instruction publique ne prendront finalement pas de risque, y compris lorsque, en 1911, c'est le félibre Maurice Faure qui a le portefeuille. Il s'en tiendra à une circulaire incitant à développer seulement l'histoire et la géographie locales à l'école primaire. Il est vrai qu'il ne restera que trois mois à son poste... Au bout du compte c'est justement dans le secondaire que le ministère accèdera à la reconnaissance de l'enseignement de la langue d'oc (à condition que les chaires ne soient pas à sa charge...), et le débat sur son usage à l'école primaire va reprendre après la $1^{\text {re }}$ guerre mondiale. On peut dire pourtant que la tendance lourde à identifier idiomes locaux et réaction politique, en somme la position de Grégoire, reste prédominante malgré un recul notable, empêchant la méthode savinienne de s'imposer.

De fait, c'est avec la loi Deixonne de 1951, d'ailleurs toujours en vigueur, que le combat mené par Savinian aboutira enfin à une autorisation de l'enseignement des langues régionales, au moment où l'arrêt de leur transmission familiale met en cause leur survie... et les moyens même de mettre en œuvre la méthode des versions, les enfants ne les connaissant plus. Mais le débat contemporain sur les écoles bilingues et l'immersion en Bretagne et ailleurs, n'est-il pas une résurgence, même dans des conditions différentes, de la difficulté française à reconnaître et assumer positivement un plurilinguisme pourtant banal à l'échelle de l'Europe et du monde?

\section{BIBLIOGRAPHIE}

AUROUZE J. : Histoire critique de la renaissance méridionale au XIX ${ }^{e}$ siècle La pédagogie régionaliste. Avignon, Seguin-Roumanille, 1907.

BELLANGER C, GODECHOT J., GUIRAL P. et TERROU F. (dir.) : Histoire générale de la Presse française (tome III, 1871-1940). Paris, PUF, 1972.

BOUTAN P. : La langue des Messieurs Histoire de l'enseignement du français à l'école primaire. Paris, Armand Colin, 1996.

BOUTAN P. : De l'enseignement des langues Michel Bréal linguiste et pédagogue. Paris, Hatier, 1998. BOUTAN P. : «Langue nationale et langues régionales à l'école : le débat politique de 1925 », in Mots Les langages du politique, N" 61, Presses de Sciences Po, décembre 1999, pp. 29-48. 
MARTEL P. : « L'école de la III République et l'occitan », in Tréma (L'école rurale, colloque de Mende), №12-13, Montpellier, IUFM de Montpellier, 1997.

MISTRAL F. neveu : « La vie, l'œuvre et la méthode du frère Savinien », in...Et nous verrons Berre..., Aix, Éd. du Feu, 1927.

SAVINIAN : « Historique de l'enseignement de la langue française par la méthode des versions provençales » [Titre manuscrit, répertorié sous LE 5792, Articles de journaux et revues, Méthode des Versions provençales-françaises, Bibliothèque municipale centrale Emile Zola de Montpellier, $2 t$ (depuis 1875 jusqu'à 1901)|.

SAVINIAN : «Principales brochures concernant la Méthode des Versions provençales françaises ", [Titre manuscrit, recueil de 26 brochures ou numéros de revues, conservé au CEDHRE, IUFM de Montpellier (de 1883 à 1911)].

\section{NOTES}

1. Préface à Recueil de versions provinciales pour l'enseignement du français publié par une Société littéraire, Deuxième partie, 1878, (np).

2. La plus grande partie des citations contenues dans cet article sont tirées de deux recueils, l'un en deux volumes foliotés et reliés, intitulé « Historique de l'enseignement de la langue française par la méthode des versions provençales ", visiblement dû au frère Savinian lui-même, constitué pour l'essentiel par des coupures de presse, récemment entré dans les collections de la bibliothèque municipale centrale Emile Zola de Montpellier (cote LE 5792) ; l'autre, sur le même sujet, avec le même auteur probable, constitué par des brochures ou numéros de revues (vingtsix au total), entré aussi très récemment dans les collections du Centre d'Etudes, de Documentation et de Recherche en Histoire de l'Education de 1TUFM de Montpellier (voir bibliographie).

3. Les statistiques considèrent que l'on a affaire à une population urbaine à partir de 2000 habitants agglomérés...

4. Voir les travaux d'Auguste Brun entre les deux guerres.

5. Les conditions diffèrent cependant, puisque l'annexion française a été conditionnée à un plébiscite local, ce qui n'aura pas lieu pour les provinces de l'Est, où d'ailleurs la frontière linguistique ne fut pas toujours respectée, en Lorraine en particulier.

6. In Recueil LE 5792, p. 1-2.

7. Ce qui explique que l'on découvrira quelques précurseurs à Savinian (voir DE BERLUC-PÉRUSSIS L. : "Frère Savinien et ses précurseurs... ", in Les écoles du Midi et la langue d'oc, choix de textes et de documents..., Montpellier, Imprimerie centrale du Midi, 1902, Recueil CEDRHE).

8. On peut s'étonner que ni Mistral ni Savinian n'aient entendu parler du livre de Bréal auparavant, alors qu'il avait fait du bruit à sa publication en 1872. Éloignement de la province, éloignement idéologique, surtout à l'époque de l'Ordre moral?

9. En fait Savinian a remplacé « patois " par « provençal ».

10. Toutes citations tirées de la préface, non paginée, du Recueil de versions... Troisième partie.

11. Commencer la rédaction d'une série de manuels par le niveau le plus élevé n'est d'ailleurs pas rare, encore aujourd'hui.

12. On a toutes les raisons de penser que c'est le texte reproduit dans le numéro d'août 1888 de la revue montpelliéraine Occitania, pp. 285 à 297 : « De l'Utilisation des dialectes provinciaux pour l'enseignement du français », signé $\mathrm{S}$, avec en exergue : «Le patois (le dialecte provincial) est le plus utile auxiliaire de l'enseignement du français ». Michel BRÉAL. Présent dans le recueil CEDRHE, № 11. 
13. Les frères publient leurs manuels avec la signature des initiales du Supérieur.

14. «L'élève qui arrive à l'école, parlant son provençal, est traité comme s'il n'apportait rien avec lui [... avec encore 〈provençal〉 substitué à 〈patois〉]», ou propos directement inspirés par lui : « [l'élève] trouvera plus de douceur à son foyer, plus de charmes et plus de grandeur à sa Province et il en aimera davantage la France [...]».

15. Cf bibliographie.

16. Bréal a fait connaître à plusieurs reprises son goût pour les proverbes qu'il invite à utiliser activement dans l'enseignement populaire.

17. Voirie prospectus de 1875.

18. P. XXXV, Grammaire provençale, de 1882, rééditée en fac-similé par Lacour, Nîmes, 1991.

19. La date précise n'est malheureusement pas claire dans le recueil LE 5792, p. 4. Savinian y réfute les déclarations du député d'Aix " ultra radical » Édouard Lockroy, qui avait dit à la Chambre : «Il existe encore ça et là certains patois ou dialectes qui sont restés comme les épaves des nationalités disparues, Eh bien ! les congrégations et l'Église s'étudient à faire revivre ces dialectes, à les conserver, à leur redonner l'existence, à empêcher la langue française de les détruire en se propageant. »

20. Le terme alors désigne l'ensemble des membres de l'enseignement public, sans distinction de hiérarchie.

21. Dans l'Étoile du Midi d'Arles, lettre datée du 21 janvier, LE 5792, p. 19. Le plaidoyer de Savinian devant sa Congrégation a déjà été évoqué plus haut, voir note 7.

22. 13 août 1888 .

23. Grammaire provençale, p. XXXV.

24. Texte cité d'après la Revue Félibrénne, 1890, p. 157.

25. Un échange en 1888 entre le tout-puissant journaliste parisien Francisque Sarcey et Mistral, à la suite d'un discours de celui-ci, fait l'objet de plusieurs pages de recension dans LE 5792.

26. L'Étendard et Le Constitutionnel du 2 septembre 1890, soit plusieurs mois après le discours de Sceaux de Bréal. LE 5792, p. 55.

27. L'observateur de Paris, août 1890.

28. Jules Simon avait été ministre de l'Instruction publique après le 4 septembre 1870 avec Thiers comme chef de gouvernement, et avait fait reconnaître le Félibrige d'utilité publique ; Bréal avait été membre de son cabinet officieux. Il se situait plus à droite que Ferry, auquel il avait fait concéder dans les programmes de morale de 1882 la mention des « devoirs envers Dieu ".

29. C'était ignorer que Mistral ne pouvait prétendre à aucun des corps représentés au Conseil, élus par leurs pairs par ailleurs.

30. Les articles parus dans la Tribune... en 1886 sont collés dans ES 5892 pp. 65 à 70, après d'autres de 1891.

31. Voir Pierre Boutan, La langue des Messieurs..., et Bibliographie.

32. L'Éclair de Paris, 11 avril 1896, d'après Les écoles du Midi et la langue d'oc, choi de textes et de documents..., op.cit, p. 51-52.

33. D'ARVAUX Gabriel : «Le mouvement fédéraliste », in La Terre de France revue fédéraliste, № 25, janvier 1893, p. 42. Revue qui fait suite à L'alliance Anti-juive... Recueil du CEDRHE.

34. Voir Bibliographie.

35. À la suite du congrès d'Avignon, cité plus haut, le bureau parisien de l'école du Félibrige avait rencontré le ministre et il s'était publiquement engagé. Voir le Manuel Général de l'Instruction Primaire, 1896, p. 457.

36. Revue catholique des institutions et du droit, février 1911, №2, pp. 127-128, recueil du CEDRHE.

37. Qu'est-ce qu'une nation?, réédité chez Pocket en 1995. 
38. Le XIXe siècle, août 1888. Journal très influent chez les instituteurs, dirigé par le francmaçon Edmont About. La revue de linguistique et de philologie comparée, dirigée par Abel Hovelacque et d'autres francs-maçons, s'est clairement prononcée contre la méthode utilisant les idiomes locaux (1880, p. 104 et p. 180), au nom de la théorie naturaliste du langage, qui réduit les langues à des êtres vivants, et en rappelant les rapports de Grégoire et de Barrère identifiant les patois à la réaction.

39. Marthe de $\mathrm{D}^{* * *}$, La langue d'oc à l'école et dans les patronages, Avignon, Aubanel, 1911, p. 14, CEDRHE.

40. Ibid.

41. C'est le cas pour Perbosc en 1890, voir l'article d'H. Terrai dans ce même numéro.

42. Bulletin théologique, scientifique et littéraire de l'Institut catholique de Toulouse, № 9, janvier 1897, p. 283, CEDRHE.

43. Voir BOUTAN P. : « La méthode Carré et la politique linguistique à l'école primaire de la IIIe' Republique vers la fin du XIXe siècle ", in Tréma, № 14 (Les manuels scolaires : aperçus historiques), Montpellier, IUFM de Montpellier, décembre 1998, pp. 13-26.

\section{RÉSUMÉS}

Savinian, frère des Écoles chrétiennes et majoral du Félibrige, proposa à la fin du XIXe siècle d'apprendre le français en faisant des versions provençales. Les réticences suscitées même chez un partisan des «patois» comme Bréal, reflétaient des obstacles pédagogiques, mais surtout un contexte idéologique de plus en plus tendu avec la montée du nationalisme, et l'enfoncement de l'Église dans son opposition à la République. Ce qui identifia majoritairement alors, malgré des soutiens républicains, apprentissage des idiomes locaux et réaction.

Savinian, Christian Brother and "majoral" in the "Félibrige" movement, at the end of the XIXth century, proposed teaching French by having students do translations from their mother tongue (provençal) into French. The hue and cry elicited by this idea even from such a partisan of the "patois" cause as Bréal, revealed the pedagogical difficulties involved, but it also underscored an ideological context made ever more tense by the rise of nationalism and the catholic church's increasingly reactionary stance in its opposition to the republic. This context resulted in the teaching of local idioms being identified with reactionary opinions.

\section{INDEX}

Keywords : Brothers of the Christian Schools, félibrige movement, french, language, langue d'oc language

Mots-clés : félibrige, français, frère des écoles chrétiennes, langue, langue d'oc

\section{AUTEUR}

PIERRE BOUTAN

IUFM de Montpellier, DIDAXIS-DIPRALANG 